\title{
Subluminal to superluminal propagation in a left-handed medium
}

\author{
S. Dutta Gupta \\ School of Physics, University of Hyderabad, Hyderabad-500046, India \\ R. Arun and G. S. Agarwal \\ Physical Research Laboratory, Navrangpura, Ahmedabad-380009, India
}

(Received 20 January 2004; published 22 March 2004)

\begin{abstract}
In this paper we report large group delays in the propagation of narrow-band pulses through a dispersive medium with both dielectric function and magnetic permeability negative. We show how the numerical results can be understood in terms of the phase time as calculated from the complex transmission amplitude. We also demonstrate superluminal passage and saturation of phase time in the same material albeit in a different spectral region.
\end{abstract}

DOI: 10.1103/PhysRevB.69.113104

PACS number(s): 78.20.Ci, 41.20.Jb, 84.40.-x

The unusual properties of the electromagnetic waves in a medium capable of possessing simultaneously negative dielectric constant $\epsilon$ and negative magnetic permeability $\mu$ were discovered by Veselago ${ }^{1}$ in late sixties. The waves in such media [referred to as left handed LH] are quite counterintuitive. For example, the direction of energy flow for a plane wave is opposite to the direction of propagation. Veselago had also discovered that the dispersion of the medium is important if the medium has to have both $\epsilon$ and $\mu$ negative. The recent experimental realization ${ }^{2,3}$ of the left-handed media has generated lot of new interest in such media and many interesting new results have been predicted. ${ }^{4-9}$ Houck et al. ${ }^{3}$ demonstrated that transmission of a microwave beam through a two-dimensional wedge obeys Snell's law with negative index of refraction. They also show the possibility of a rectangular slab focusing power from a single point source thus providing an almost confirmation of a prediction of Pendry ${ }^{4,5}$ that a left-handed medium under suitable conditions can become a perfect lens. Many other interesting theoretical proposals regarding electromagnetic wave propagation in a LH medium have been made. ${ }^{6-9}$ Li et al. ${ }^{7}$ show the possibility of a new type of band in a stack formed from left handed and the usual materials. Effects of material anisotropy have been considered by Smith and Schurig. ${ }^{8}$ By doing a time dependent calculation, Foteinopoulou et al. ${ }^{9}$ shed light on how the direction of wave front reverses.

As noted earlier, the dispersion of both $\epsilon$ and $\mu$ is important in LH medium. Since the dispersion is important, one should examine how the propagation of a pulse in a $\mathrm{LH}$ medium takes place. This is the object of the present study. Note that the pulse propagation through a dispersive medium has acquired a new dimension due to the possibility of producing slow light. ${ }^{10,11}$ We also note that pulses in dispersive medium have been extensively studied since the early work of Sommerfeld and Brillouin and a large body of literature exists. ${ }^{12-14}$ In what follows we concentrate on the specific aspects of pulses in LH media and report large group delays in the propagation of narrow-band pulses. We also demonstrate superluminal passage and saturation of phase time in the same material albeit in a different spectral region. We thus realize analog of Hartman effect ${ }^{15,16}$ well known in quantum-mechanical tunneling problems. The numerical re- sults can be understood in terms of the phase time ${ }^{17}$ as calculated from the complex transmission amplitude.

Consider a monochromatic wave with frequency $\omega$ to be incident normally on a parallel slab of the medium exhibiting negative refractive index as shown in Fig. 1. Let $\epsilon(\omega), \mu(\omega)$ be the frequency-dependent material parameters and both are negative simultaneously for a negative-refraction medium. The plane-wave solutions for the electric-field vector $\vec{E}(z, t)=E(z) \exp (-i \omega t) \hat{y}$ in different regions are given by

$$
\begin{gathered}
E(z)=A \exp (i k z)+B \exp (-i k z), \quad z \leqslant 0, \\
=C \exp (i q z)+D \exp (-i q z), \quad 0 \leqslant z \leqslant d, \\
=F \exp [i k(z-d)], \quad z \geqslant d,
\end{gathered}
$$

with $k=\omega / c$ and $q=(\omega / c) \sqrt{\epsilon(\omega) \mu(\omega)}$. For the LH medium the term $D e^{-i q z}\left(C e^{i q z}\right)$ corresponds to transport of energy in the positive (negative) $z$ direction. Note that for a plane wave with $\vec{E} e^{i \vec{k} \cdot \vec{r}}$, the magnetic field will depend on $\mu$ through the relation $\bar{H}=(c / \mu \omega)(\vec{k} \times \vec{E}) e^{i \vec{k} \cdot \vec{r}}$. The transmission amplitude $F$ can be obtained by using the boundary conditions at the interface $z=0$ and $z=d$. We use the continuity of the tangential components of the electric and magnetic fields to find the result

$$
\begin{aligned}
T(\omega) & =F / A=|T(\omega)| \exp [i \phi(\omega)] \\
& =\left[\cos (q d)-\frac{i}{2}\left(\frac{q}{\mu k}+\frac{\mu k}{q}\right) \sin (q d)\right]^{-1} .
\end{aligned}
$$

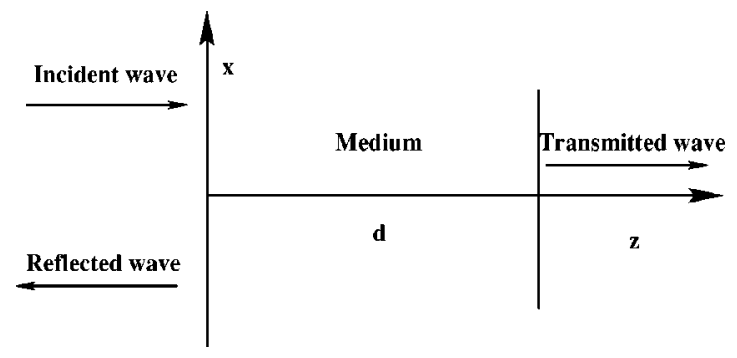

FIG. 1. Incident, reflected, and transmitted waves in a slab of LH/RH medium of width $d$. 


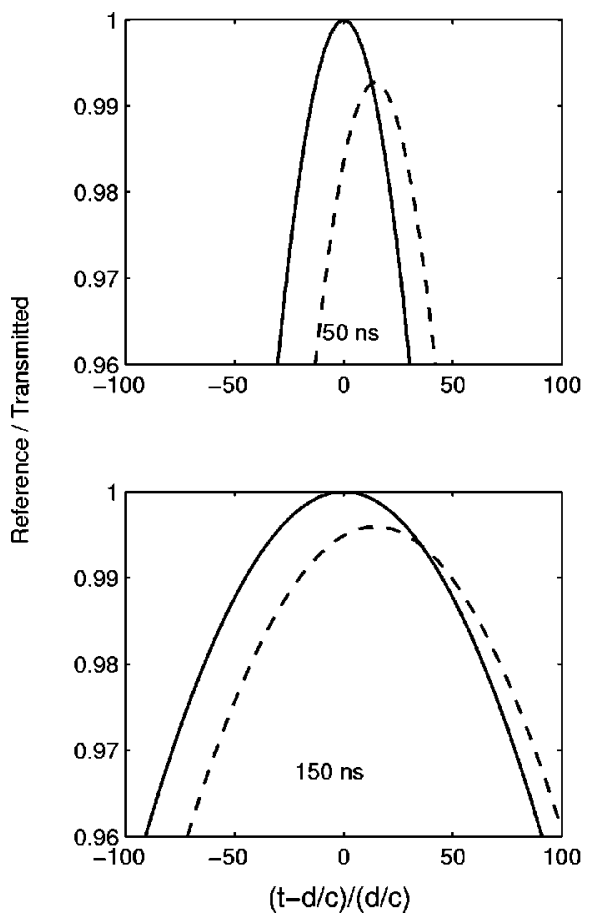

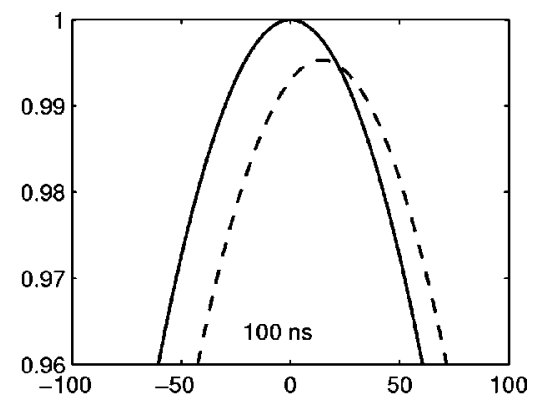

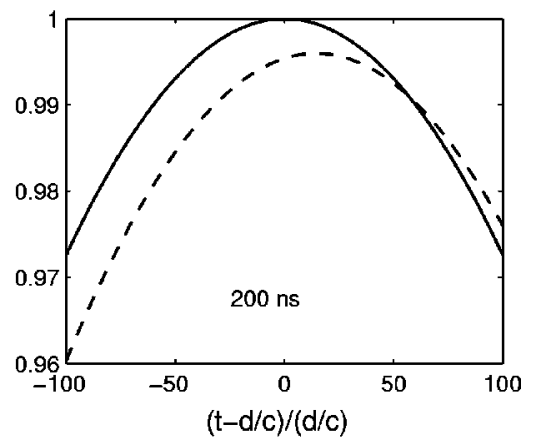

FIG. 2. Reference (solid) and transmitted (dashed) pulse temporal profiles for carrier frequency $f_{l}=5 \mathrm{GHz}, d=10 \mathrm{~cm}$. Different curves are labeled by different values of $\tau$, namely, $\tau=50,100$, 150 , and $200 \mathrm{~ns}$.
Clearly the phase of the transmitted amplitude depends whether one is dealing with propagation in a LH or righthanded RH medium. It is also interesting to note that the natural modes of oscillation and their frequencies of the system depend on the handedness of the medium. By setting the denominator in Eq. (2) zero, one gets these frequencies.

We now consider the propagation of a narrow-band pulse through the medium. The incident field can be decomposed into its Fourier components

$$
E(z, t)=\int_{-\infty}^{\infty} d \omega A(\omega) e^{-i \omega t+i(\omega / c) z}
$$

We assume that $A(\omega)$ is sharply peaked around a central frequency $\omega_{l}$. Using Eqs. (1) and (2), the transmitted pulse will be given by

$$
E_{T}(z, t)=\int_{-\infty}^{\infty} d \omega A(\omega)|T(\omega)| e^{i \phi(\omega)-i \omega t} \exp \left\{\frac{i \omega}{c}(z-d)\right\}
$$

Thus the behavior of the output pulse can be analyzed by numerical evaluation of the integral (4). We consider the case of a Gaussian pulse

$$
\begin{gathered}
E(z, t)=e^{-i \omega_{l} \bar{t}} \exp \left\{-(\bar{t} / \tau)^{2}\right\}, \quad \bar{t}=t-\frac{z}{c}, \\
A(\omega)=\frac{\tau}{2 \sqrt{\pi}} \exp \left\{-\left(\omega-\omega_{l}\right)^{2} \frac{\tau^{2}}{4}\right\} .
\end{gathered}
$$

For material parameters we use the values used by Smith and $\mathrm{Kroll}^{6}$

$$
\epsilon(\omega)=\frac{\left(\omega^{2}-\omega_{p}^{2}\right)}{\omega^{2}}, \quad \mu(\omega)=\frac{\left(\omega^{2}-\omega_{b}^{2}\right)}{\left(\omega^{2}-\omega_{o}^{2}\right)},
$$

which are to be understood as the zero-damping limit of full expressions. In Eq. (7), $\omega_{p, b, o}=2 \pi f_{p, b, o}$ with $f_{p}=12 \mathrm{GHz}$, $f_{b}=6 \mathrm{GHz}$, and $f_{o}=4 \mathrm{GHz}$. The refractive index will be positive for $\omega>\omega_{p}$ and negative for $\omega_{o}<\omega<\omega_{b}$. In other ranges of $\omega$, the refractive index will be purely imaginary and the corresponding waves will represent the evanescent modes. The numerical results for the output (transmitted) pulses are shown in the Fig. 2. We have verified, that for the parameters of Fig. 2, the output pulses are Gaussian. For comparison, we have also shown the reference pulse, i.e., a pulse that travels the distance $d$ in free space. The carrier frequency $f_{l}$ was chosen at the center of LH band, i.e., at 5 GHz. Calculations were carried out for $d=10 \mathrm{~cm}$ and for four different values of $\tau$, namely, $\tau=50,100,150$, and 200 ns. Note that the pulse delays by about $5.2 \mathrm{~ns}$ and the delay is more or less independent of the pulse width. Figure 2 clearly demonstrates the possibility of significant pulse delays in a LH medium. These pulse delays can be understood by considering the phase time. ${ }^{17,18}$ It is essentially the time by which the peak of the transmitted pulse is delayed. The integral of the transmitted field in Eq. (4) can be evaluated approximately by making the Taylor expansion of the phase $\phi(\omega)$ of transmission amplitude about the carrier frequency $\omega_{l}$ of the pulse. Keeping terms up to first order in the expansion and assuming the narrow-band approximation $|T(\omega)|$ $\approx\left|T\left(\omega_{l}\right)\right|$, one can show that pulse delay is given by the phase time defined as

$$
t_{p h}=\left.\frac{\partial \phi(\omega)}{\partial \omega}\right|_{\omega=\omega_{l}}
$$



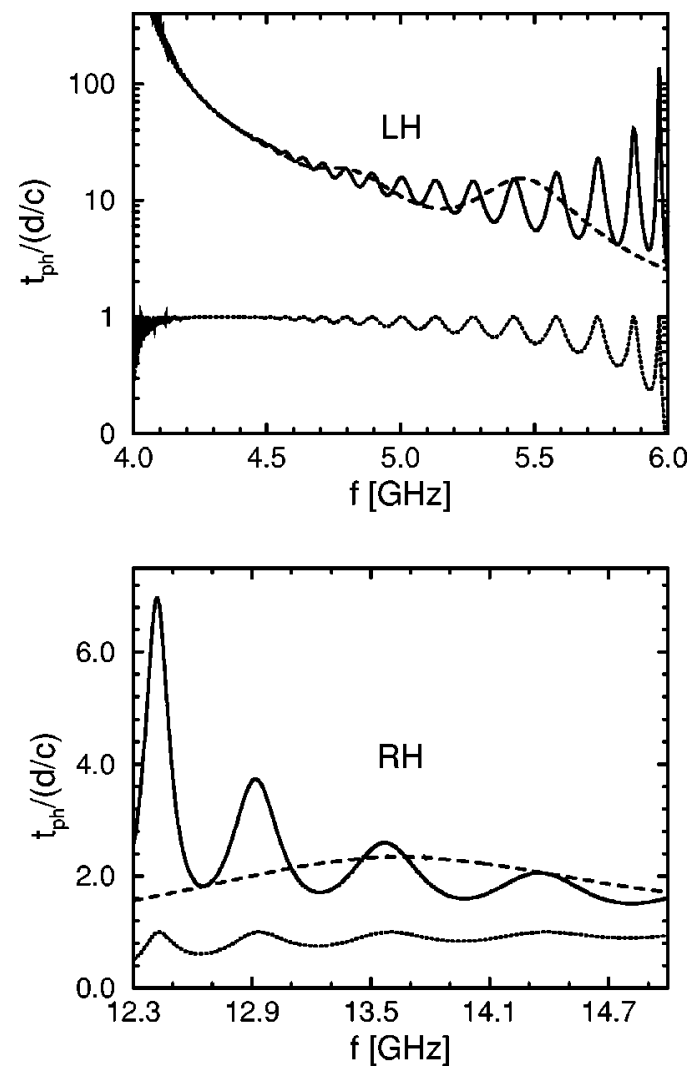

FIG. 3. Normalized phase time for the LH (top) and RH (bottom) media for $d=10 \mathrm{~cm}$ (solid) and $2 \mathrm{~cm}$ (dashed), respectively. The dotted curves represent the modulus of transmission amplitude $T(\omega)$ for a fixed medium width $d=10 \mathrm{~cm}$.

The phase time $t_{p h}$ can be calculated from the transmission amplitude $T(\omega)$ remembering that $\mu$ and $q$ are functions of $\omega$. On carrying out the differentiation in Eq. (8), we get the result for the phase time in the form

$$
\begin{gathered}
t_{p h} \equiv \frac{N}{D} \\
N=\frac{1}{2} \tan [\omega d m(\omega) / c]\left(\frac{1}{\mu} \frac{\partial m}{\partial \omega}-\frac{\mu}{m^{2}} \frac{\partial m}{\partial \omega}-\frac{m}{\mu^{2}} \frac{\partial \mu}{\partial \omega}+\frac{1}{m} \frac{\partial \mu}{\partial \omega}\right) \\
+\frac{1}{2}\left(\frac{m}{\mu}+\frac{\mu}{m}\right) \sec ^{2}[\omega d m(\omega) / c] \frac{\partial}{\partial \omega}[\omega m(\omega)] \frac{d}{c} \\
D=1+\frac{1}{4}\left(\frac{m}{\mu}+\frac{\mu}{m}\right)^{2} \tan ^{2}[\omega d m(\omega) / c] \\
m(\omega) \equiv \sqrt{\epsilon(\omega) \mu(\omega)} .
\end{gathered}
$$

The phase time (9) is to be compared with the time $t_{o}$ $=d / c$ for the free-space propagation. The numerical results for the phase time are shown in the Fig. 3. The top (bottom) curve is for the LH (RH) medium. The solid (dashed) lines are for $d=10 \mathrm{~cm}(2 \mathrm{~cm})$. It is clear from Fig. 3 that the LH medium leads to very large group delays and therefore to slow light. The results for the ratio of the delays (about 16)
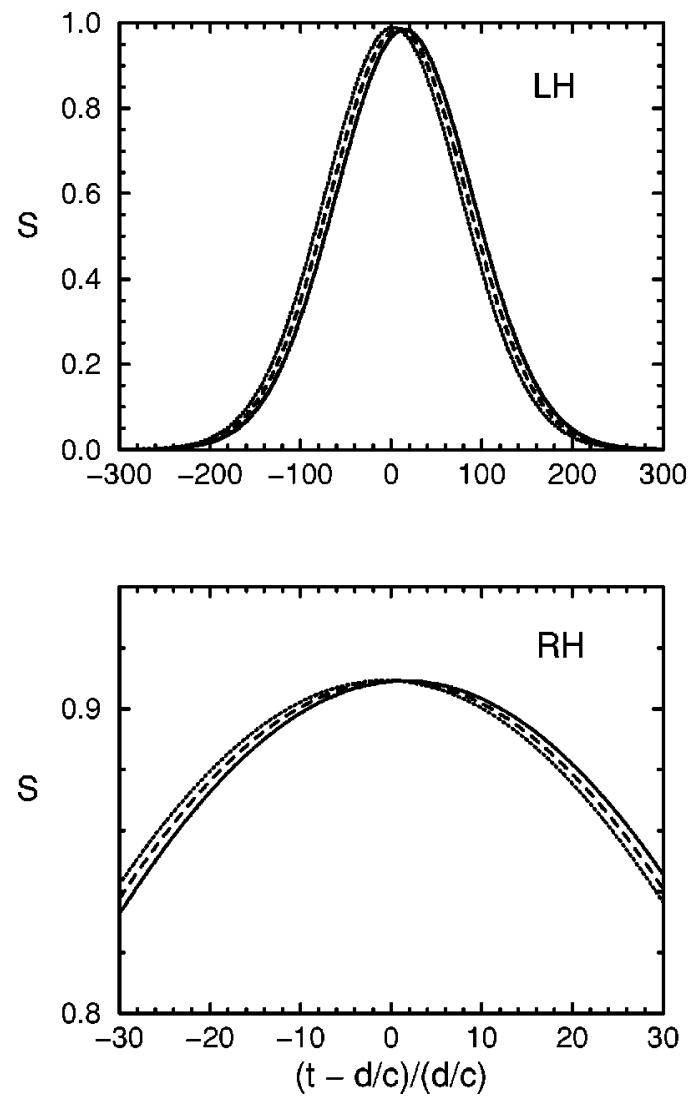

FIG. 4. Temporal evolution of the pulse intensity $S$ $\equiv \operatorname{Re}\left[E(z, t) \times H^{*}(z, t)\right]$ at different spatial locations inside the medium. The parameters for the calculations are $\tau=50 \mathrm{~ns}, d$ $=10 \mathrm{~cm}$, and $f_{l}=5 \mathrm{GHz}(\mathrm{LH}), f_{l}=13.5 \mathrm{GHz}(\mathrm{RH})$. The dotted, dashed, and solid curves correspond to $z=2 \mathrm{~cm}, z=6 \mathrm{~cm}$, and $z$ $=10 \mathrm{~cm}$, respectively.

for a carrier at $5 \mathrm{GHz}$ and $d=10 \mathrm{~cm}$ is in conformity with those of Fig. 2. The role of the slab resonances ${ }^{19,20}$ can also be seen from Fig. 3. The resonances are determined by the poles of the transmission amplitude and therefore depend on the nature of the medium. The poles differ significantly for the LH and RH media, as can be seen in Fig. 3. We note that, the resonance nature of the pulse delay here is very similar to those studied by Kuzmiak and Maradudin for spheres. ${ }^{21}$ Further, Fig. 3. also shows that, in a very thin slab $(d=2 \mathrm{~cm})$, one can achieve group index similar to that of a thicker slab $(d=10 \mathrm{~cm})$ by means of the resonance enhancement (see comparable values of phase times close to $f=4.8 \mathrm{GHz}$ in Fig. 3). For $d=2 \mathrm{~cm}$, a time delay of $5 \mathrm{~ns}$ would correspond to the group index of 75. A group index of this order is quite significant for solid-state systems. Our calculated group index is close to the experimental value of Parimi et al. ${ }^{22}$ They have obtained a group index of the order of 100 in a waveguide filled with left-handed materials. The group indices for LH media are much larger than for other solid-state systems like Fibonacci layer, for which values of less than 10 have been reported. ${ }^{23}$ We mention further, that the group delays of transmitted pulse in Fig. 2 are a consequence of multiple reflections of waves at the boundaries of the medium. Recall that we are dealing with a slab and there are both forward 


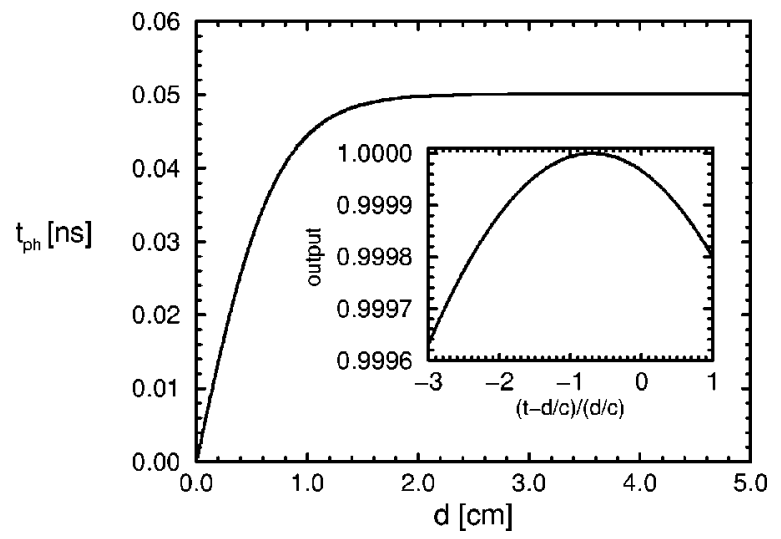

FIG. 5. The phase time as a function of width $d$ of the medium for a fixed frequency $\omega_{l}=(2 \pi) 9 \mathrm{GHz}$. The inset shows the temporal profile of the transmitted pulse for a fixed width $d=5 \mathrm{~cm}$ and $\tau=20 \mathrm{~ns}$. Actual values of the curve in the inset are 0.00198 times those shown and its peak occurs at $t_{p h}=0.3 \mathrm{~d} / \mathrm{c}$ given by the expression (9) of phase time.

and backward waves inside the medium. ${ }^{24}$ To understand the nature of pulse delays, we have also plotted in Fig. 4 the real part of $\vec{E}(z, t) \times \vec{H}^{*}(z, t)$ which gives the magnitude of the Poynting vector at different points inside the medium. The graph shows that the energy propagates slower through the LH medium in comparison with its traversal through RH medium. Also, there is always a net flow of energy in the positive $z$ direction.

We have so far considered pulse propagation in spectral regions, where, irrespective of the sign, the refractive index is real and hence the waves are of propagating nature. In both the cases, the medium slows down the pulse in transmission. Interestingly, in the intermediate domain $\left(\omega_{b}<\omega<\omega_{p}\right)$, one can have superluminal tunneling of the pulse. Note that in this region, the material exhibits a metallike behavior [since $\epsilon(\omega)<0, \mu(\omega)>0$ ] and a slab of such a material acts like a typical "barrier." In Fig. 5, we show the behavior of phase time as a function of the medium width $d$ when the carrier frequency of the pulse lies at the center of the evanescent region $\omega_{b}<\omega<\omega_{p}$. The phase time is seen to saturate as a function of the medium width and it is less than the freespace traversal time $d / c$ as shown in the inset for the transmitted pulse. The saturation of phase time with medium width, known as "Hartman effect" in the literature, ${ }^{15}$ can be understood from the analytical formula, Eq. (9), with purely imaginary values of $m$ corresponding to the evanescent modes. Recently, the Hartman effect and the superluminal tunneling of pulses have been given a new interpretation in terms of an energy storage inside the medium. ${ }^{16}$

In conclusion we have demonstrated that the LH media could give rise to large pulse delays and these delays can be understood in terms of the phase time defined via the phase of the transmission amplitude. The use of such materials in resonators is expected to enhance group delays which might be useful for the development of delay lines (cf. Ref. 20). We further showed how the necessary dispersion of the LH medium also enables us to realize superluminal propagation.

One of us (G.S.A.) thanks J. H. Eberly for a discussion of the Hartman effect.
${ }^{1}$ V.G. Veselago, Sov. Phys. Usp. 10, 509 (1968).

${ }^{2}$ R.A. Shelby, D.R. Smith, S.C. Nemat-Nasser, and S. Schultz, Appl. Phys. Lett. 78, 489 (2001); R.A. Shelby, D.R. Smith, and S. Schultz, Science 292, 77 (2001).

${ }^{3}$ A.A. Houck, J.B. Brock, and I.L. Chuang, Phys. Rev. Lett. 90, 137401 (2003).

${ }^{4}$ J.B. Pendry, Phys. Rev. Lett. 85, 3966 (2000); G.W. 't Hooft, ibid. 87, 249701 (2001).

${ }^{5}$ G. Gómez-Santos, Phys. Rev. Lett. 90, 077401 (2003).

${ }^{6}$ D.R. Smith and N. Kroll, Phys. Rev. Lett. 85, 2933 (2000).

${ }^{7}$ Jensen Li, Lei Zhou, C.T. Chan, and P. Sheng, Phys. Rev. Lett. 90, 083901 (2003).

${ }^{8}$ D.R. Smith and D. Schurig, Phys. Rev. Lett. 90, 077405 (2003).

${ }^{9}$ S. Foteinopoulou, E.N. Economou, and C.M. Soukoulis, Phys. Rev. Lett. 90, 107402 (2003).

${ }^{10}$ L.V. Hau, S.E. Harris, Z. Dutton, and C.H. Behroozi, Nature (London) 397, 594 (1999).

${ }^{11}$ M.M. Kash, V.A. Sautenkov, A.S. Zibrov, L. Hollberg, G.R. Welch, M.D. Lukin, Y. Rostovtsev, E.S. Fry, and M.O. Scully, Phys. Rev. Lett. 82, 5229 (1999); M.S. Bigelow, N.N. Lepeshkin, and R.W. Boyd, ibid. 90, 113903 (2003).
${ }^{12}$ C.G.B. Garrett and D.E. McCumber, Phys. Rev. A 1, 305 (1970).

${ }^{13}$ K. E. Oughstun, Electromagnetic Pulse Propagation in Causal Dielectrics (Springer-Verlag, New York, 1994).

${ }^{14}$ M. Scalora et al., Phys. Rev. E 54, R1078 (1996).

${ }^{15}$ T.E. Hartman, J. Appl. Phys. 33, 3427 (1962).

${ }^{16}$ H.G. Winful, Opt. Express 10, 1491 (2002); H.G. Winful, Phys. Rev. Lett. 90, 023901 (2003).

${ }^{17}$ E.P. Wigner, Phys. Rev. 98, 145 (1955).

${ }^{18}$ R. Y. Chiao and A. M. Steinberg, in Progress in Optics, edited by E. Wolf (Elsevier, Amsterdam, 1997), Vol. 37, p. 345.

${ }^{19}$ Pedro Pereyra, Phys. Rev. Lett. 84, 1772 (2000).

${ }^{20}$ J.E. Heebner and R.W. Boyd, J. Mod. Opt. 49, 2629 (2002).

${ }^{21}$ V. Kuzmiak and A. Maradudin, Phys. Rev. B 66, 045116 (2002).

${ }^{22}$ P. V. Parimi, P. Vodo, W. T. Lu, S. Sridhar, and J. S. Derov (private communication).

${ }^{23}$ Luca Dal Negro et al., Phys. Rev. Lett. 90, 055501 (2003).

${ }^{24}$ One can also study the behavior of reflected pulse at the entry face $(z=0)$ of the slab instead of the transmitted pulse. The pulse delay of the reflected fields can be shown to be equal to that of the transmitted pulse, as discussed in Ref. 16. 